\title{
Association of pro-inflammatory soluble cytokine receptors early during hepatocellular carcinoma stereotactic radiotherapy with liver toxicity
}

\author{
Sylvia S. W. $\mathrm{Ng}^{1,2}$, Hong Zhang $\mathbb{D}^{3}$, Lisa Wang ${ }^{4}$, Deborah Citrin (iD $^{3}$ and Laura A. Dawson ${ }^{1,2 \bowtie}$
}

Plasma levels of soluble factors early during hepatocellular carcinoma (HCC) stereotactic body radiotherapy (SBRT) were evaluated in relation to radiation liver injury, tumor response, and risk of early death. No significant differences were found in baseline plasma levels of AFP, CXCL1, and HGF amongst HCC patients with different Child Pugh scores. Higher levels of sTNFRII $(P<0.001)$, and lower levels of SCD40L $(P<0.001)$ and CXCL1 $(P=0.01)$ following one to two fractions of SBRT were noted in patients who developed liver toxicity vs. those who did not. High circulating levels of AFP (HR 2.16, $P=0.04$ ), sTNFRII (HR 2.27, $P=0.01)$, and sIL-6R (HR 1.99, $P=0.03$ ) early during SBRT were associated with increased risk of death 3 months post treatment. Plasma levels of the studied factors early during SBRT were not associated with tumor response. A pro-inflammatory systemic environment is associated with development of liver toxicity and increased risk of early death following SBRT.

npj Precision Oncology (2020)4:17; https://doi.org/10.1038/s41698-020-0124-z

\section{INTRODUCTION}

Stereotactic body radiotherapy (SBRT) refers to the use of high doses of radiation per fraction in fewer fractions with high precision and accuracy to achieve local tumor control. For advanced hepatocellular carcinoma (HCC) patients who are not candidates for local therapies, such as surgery, radiofrequency ablation, or transcatheter arterial chemoembolization, liver SBRT is a treatment option. Prior clinical studies have demonstrated that liver SBRT is well tolerated and efficacious, with 1-year and 3-year local control rates of $95 \%$ and $85 \%$, respectively, in Child Pugh A HCC patients ${ }^{1-4}$. Child Pugh B HCC patients have also been treated effectively with liver SBRT, although with an increased risk of toxicity ${ }^{5}$. Child Pugh score is routinely used as a surrogate of liver function in patients with chronic liver diseases and to stratify HCC patients who are in clinical trials ${ }^{6}$. More recently, albumin-bilirubin (ALBI) grade has been shown to be a more objective and discriminatory metric for the assessment of liver function in HCC patients ${ }^{7}$.

The mechanisms of action of SBRT are thought to be different from those of conventional fractionation radiotherapy upon which the classical radiobiological principles of repopulation, repair, redistribution, and reoxygenation were founded. SBRT has been shown in preclinical studies to induce secondary tumor cell death by causing tumor-associated endothelial cell death ${ }^{8,9}$ and vascular damage ${ }^{8,10}$, in addition to direct tumor cell kill by generating DNA strand breaks. Furthermore, preclinical data also suggested that SBRT-induced massive tumor cell death triggers the release of tumor antigens and inflammatory cytokines, thereby stimulating anti-tumor immune response ${ }^{8,11}$. The mechanisms by which SBRT confers tumor control or causes acute/late normal tissue toxicity in the clinical setting remain to be elucidated.

It has been suggested that radiation modulates the immune system within the tumor microenvironment and in the systemic circulation in several ways ${ }^{11}$. For instance, radiation releases a group of tumor antigens and molecules, collectively known as damage-associated molecular patterns (DAMPS), after tumor cell kill, inducing the expression of immunomodulatory cytokines and membrane-bound/soluble cytokine receptors amongst others and contributing to a pro-inflammatory local and systemic environment $^{11}$. Radiation also increases tumor vascular permeability, causing increased extravasation of antigen-presenting cells and effector T cells ${ }^{11}$. Whether immunosuppression or immunostimulation prevails following radiotherapy partially depends on the levels of circulating, paracrine, and autocrine immunomodulatory factors as well as immune cells. To further complicate the picture, many immunomodulatory cytokines have receptors that are in membrane-bound and soluble forms. Binding of the ligand to its respective membrane-bound or soluble receptors can elicit agonistic or antagonistic effects of the ligand. While the circulating levels of various cytokines have been reported in HCC patients in the literature, we are not aware of any published studies that examine the levels of soluble cytokine receptors as a group in HCC patients at baseline and during SBRT.

The specific aims of our study are first, to determine whether the circulating levels of a panel of soluble cytokine receptors and liver-secreted proteins in HCC patients are associated with the degree of liver impairment at baseline and early during SBRT; and second, to assess if the plasma levels of these soluble factors following one to two fractions of SBRT are associated with the ultimate development of radiation-induced liver injury, radiographic tumor response, and risk of death at 3 months after completion of treatment.

\section{RESULTS}

The present study included 38 of 102 Child Pugh A and 9 of 29 Child Pugh B patients from two previous published prospective clinical trials ${ }^{1,5}$. These 47 patients had plasma samples collected at

\footnotetext{
${ }^{1}$ Radiation Medicine Program, Princess Margaret Cancer Centre, Toronto, ON, Canada. ${ }^{2}$ Department of Radiation Oncology, University of Toronto, Toronto, ON, Canada. ${ }^{3}$ Radiation Oncology Branch, Centre for Cancer Research, National Cancer Institute, National Institutes of Health, Bethesda, MD, USA. ${ }^{4}$ Department of Biostatistics, Princess Margaret Cancer Centre, Toronto, ON, Canada. ${ }^{凶}$ email: Laura.Dawson@rmp.uhn.on.ca
} 
baseline and after having received one to two of six fractions of SBRT. The patient and treatment characteristics are shown in Table 1. Frequency bias was noted for gender with $79 \%$ of patients being male, Child Pugh score with $81 \%$ of patients being Child Pugh $A$, and ALBI grade with $6 \%$ of patients being grade 3 . In-field tumor response at 3 months post SBRT was available for 31 of 38 Child Pugh A patients, and 6 of 9 Child Pugh B patients due to death or absence of imaging. Regarding in-field tumor response, $57 \%$ of patients had stable disease, while $41 \%$ of patients demonstrated a partial response at 3 months post SBRT. There was frequency bias with only $3 \%(n=1)$ of patients having a complete response to SBRT at 3 months.

The plasma levels of 19 of the 28 plasma soluble factors being analyzed were not within the detection range of the assays and therefore could not be quantified. The nine factors whose plasma levels were within the range of detection of the assays included sCD40L, sTNFRII, sIL-6R, AFP, ANGPTL4, CXCL1, HGF, sEGFR, and sgp130. Furthermore, the plasma levels of 1,7 , and 18 of the 47 patient samples were not within the detection range of the assays for ANGPTL4, AFP, and HGF, respectively.

At baseline, significant differences were observed in the plasma levels of sTNFRII $(P=0.002)$, ANGPTL4 $(P=0.024)$, sgp130 ( $P=$ $0.002)$, and sEGFR $(P=0.013)$ amongst Child Pugh 5, 6, and 7 HCC patients (Fig. 1). When HCC patients were stratified by ALBI grade, significant differences were also detected in the plasma levels of sCD40L $(P=0.02)$, sTNFRII $(P=0.003)$, ANGPTL4 $(P=0.018)$, and sgp130 $(P<0.001)$ amongst the grade 1,2 , and 3 cohorts (Fig. 2$)$. The higher the Child Pugh score or ALBI grade, the higher the plasma levels of sTNFRII, ANGPTL4, and sgp130 were noted (Figs. 1, 2). In contrast, sCD40L levels decreased with increasing Child Pugh score. Since SCD40L is primarily shed by activated platelets in cancer patients ${ }^{12,13}$, we evaluated the association between plasma sCD40L levels and platelet number. Plasma sCD40L levels were found to be positively associated with platelet number $(\rho=$ $0.529, P=0.0001)$ at baseline. There were no significant differences in the baseline plasma levels of sIL-6R, AFP, CXCL1, and HGF amongst Child Pugh 5, 6, and 7 or ALBI grade 1, 2, and 3 HCC patients. Furthermore, there were no significant differences, when the levels of each tested soluble factor per cc tumor volume were examined.

Following only one to two of the planned six fractions of SBRT, significant differences were noted in the plasma levels of sTNFRII $(P=0.006)$, ANGPTL4 $(P=0.02)$, and sgp130 $(P=0.008)$ but not sEGFR $(P=0.22)$ amongst Child Pugh 5, 6, and 7 HCC patients (Fig. 1). When HCC patients were stratified by ALBI grade, there were significant differences in the plasma levels of sCD40L $(P=$ $0.01)$, sTNFRII $(P=0.007)$, and sgp130 $(P=0.007)$, but not ANGPTL4 $(P=0.06)$, sIL-6R $(P=0.60)$, and sEGFR $(P=0.69)$ amongst the grade 1, 2, and 3 cohorts (Fig. 2). Plasma SCD40L levels were also noted to be positively associated with platelet number ( $\rho=0.524, P=0.0002$ ) after one to two fractions of SBRT. No significant differences were found in the plasma levels of AFP, CXCL1, and HGF amongst Child Pugh 5, 6, and 7 or ALBI grade 1, 2, and 3 HCC patients.

We next asked whether the levels of plasma soluble factors following only one to two of the planned six fractions of SBRT could shed light on the presence/absence of liver toxicity 3 months after completion of SBRT. As shown in Fig. 3, significantly higher levels of sTNFRII $(P<0.001)$ as well as lower levels of sCD40L $(P<$ $0.001)$ and $\operatorname{CXCL1}(P=0.01)$ after one to two fractions of SBRT were noted in HCC patients who developed liver toxicity as reflected by $\geq 2$ points decline in Child Pugh score vs. those who did not at 3 months after SBRT. Similar plasma levels of ANGPTL4, sgp130, AFP, and HGF following only one to two fractions of SBRT were detected in HCC patients with or without liver toxicity at 3 months post SBRT.

As shown in Table 2, the plasma levels of sCD40L, sTNFRII, sIL6R, AFP, ANGPTL4, CXCL1, HGF, sEGFR, and sgp130 early during
Table 1. Patient and treatment characteristics.

\begin{tabular}{|c|c|}
\hline Variable & Number of patients (\%) \\
\hline Age, median (range) & $70(48-90)$ \\
\hline \multicolumn{2}{|l|}{ Gender } \\
\hline Male & 37 (79\%) \\
\hline Female & $10(21 \%)$ \\
\hline \multicolumn{2}{|l|}{ Cause of underlying chronic liver disease } \\
\hline Alcohol & $22(47 \%)$ \\
\hline Hepatitis B & $13(28 \%)$ \\
\hline Hepatitis C & $22(47 \%)$ \\
\hline Non-alcoholic steatohepatitis & $2(4 \%)$ \\
\hline \multicolumn{2}{|l|}{ Child Pugh score } \\
\hline A5 & 27 (57\%) \\
\hline A6 & $11(24 \%)$ \\
\hline B7 & $9(19 \%)$ \\
\hline \multicolumn{2}{|l|}{$A L B I$ grade } \\
\hline 1 & $20(43 \%)$ \\
\hline 2 & $24(51 \%)$ \\
\hline 3 & $3(6 \%)$ \\
\hline \multicolumn{2}{|l|}{ HCC thrombus } \\
\hline Absent & $21(45 \%)$ \\
\hline Present & $26(55 \%)$ \\
\hline \multicolumn{2}{|l|}{ Previous treatment } \\
\hline Any & $23(49 \%)$ \\
\hline None & $24(51 \%)$ \\
\hline \multicolumn{2}{|l|}{ In-field tumor response at 3 months } \\
\hline Stable disease & $21(57 \%)$ \\
\hline Partial response & $15(41 \%)$ \\
\hline Complete response & $1(2 \%)$ \\
\hline \multicolumn{2}{|l|}{ Baseline laboratory values, median (range) } \\
\hline Bilirubin, $\mu \mathrm{mol} / \mathrm{L}$ & $14(6-42)$ \\
\hline Albumin, $\mathrm{g} / \mathrm{L}$ & $38(23-47)$ \\
\hline Platelet, $\times 10^{9} / \mathrm{L}$ & $117(55-366)$ \\
\hline Gross tumor volume, mL, median (range) & $96.8(1.3-1385.1)$ \\
\hline Prescription dose, Gy, median (range) & $33(30-54)$ \\
\hline Liver mean dose, Gy, median (range) & $15.3(4.3-18.2)$ \\
\hline D800cc, Gy, median (range) & $7.4(0-25.3)$ \\
\hline Veff, \%, median (range) & $39(9-60)$ \\
\hline Liver volume, mL, median (range) & $1231.4(750-3080.7)$ \\
\hline
\end{tabular}

SBRT were not associated with in-field tumor response at 3 months after completion of SBRT.

The median level of each soluble factor for all patients was used for stratification and analysis with the Cox proportional hazard model to determine if <median (low) vs. $\geq$ median (high) level of each soluble factor at baseline and early during SBRT was associated with increased or decreased risk of death at 3 months post SBRT. As shown in Table 3, high baseline levels of SCD40L were significantly associated with lower risk of death (HR 0.52, $95 \% \mathrm{Cl} 0.27-0.99, P=0.05)$. In contrast, high baseline levels of sTNFRII (HR 1.93, 95\% Cl 1.02-3.65, $P=0.04)$, sIL-6R (HR 1.9, 95\% Cl $1.01-3.57, P=0.05)$, AFP (HR 2.16, 95\% Cl 1.03-4.54, $P=0.043$ ), sEGFR (HR 2.61, 95\% Cl 1.32-5.16, $P=0.006$ ), and sgp130 (HR 2.19, $95 \% \mathrm{Cl} 1.13-4.25, P=0.021)$ were significantly associated with higher risk of death. There was no significant association between high or low baseline levels of ANGPTL4, CXCL1, and HGF with the risk of early death (Table 3). After one to two fractions of SBRT, only high circulating levels of AFP (HR 2.16, 95\% Cl 1.03-4.54, 

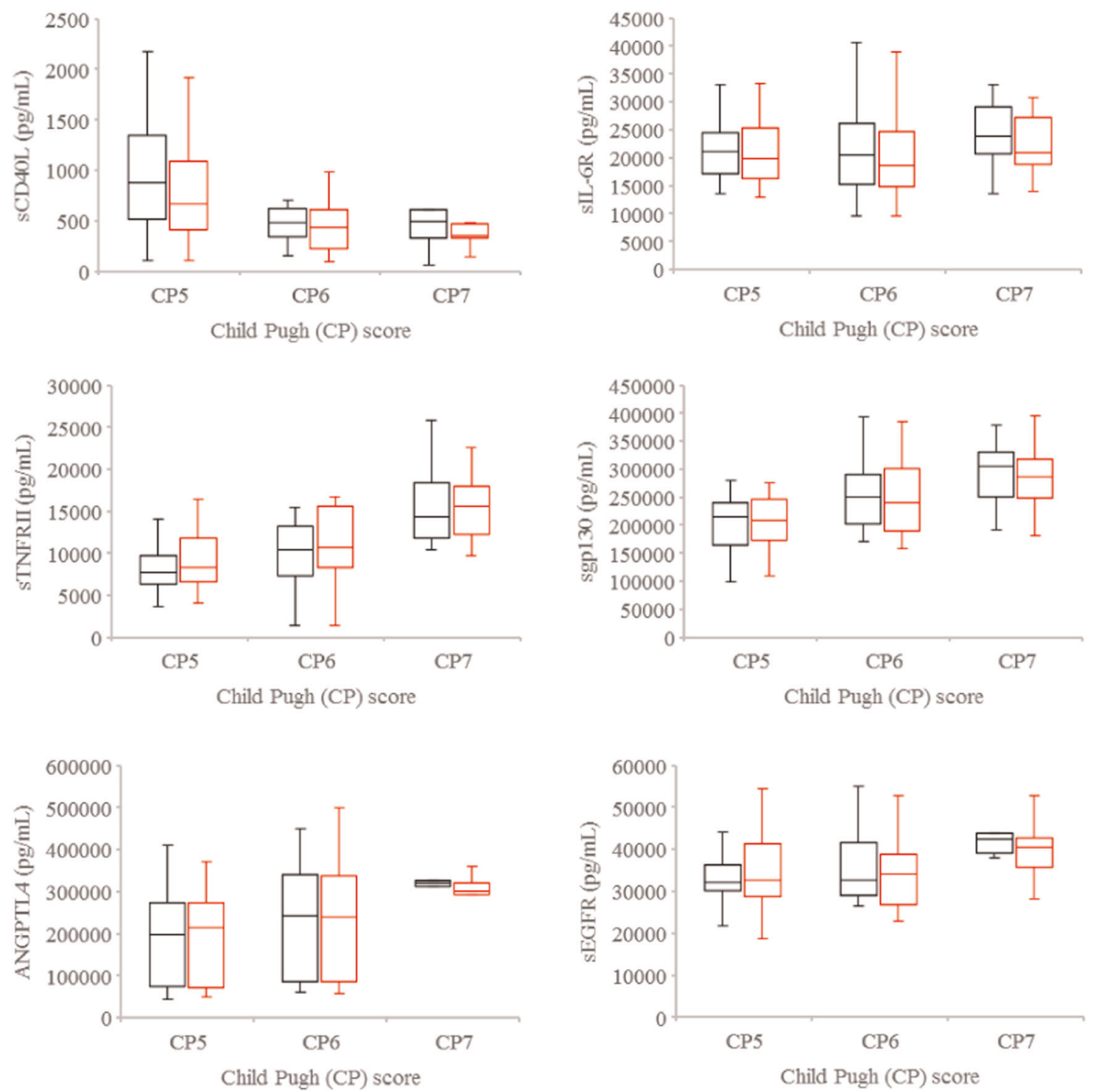

Fig. 1 Plasma levels of soluble factors by Child Pugh scores. Boxplots of plasma levels of sCD40L, sTNFRII, ANGPTL4, sIL-6R, sgp130, and sEGFR in Child Pugh 5, 6, and 7 HCC patients at baseline (black) and after one to two fractions of SBRT (red). Horizontal lines indicate median values, 25th-75th percentiles; whiskers indicate standard deviation.

$P=0.04)$, sTNFRII (HR 2.27, 95\% Cl 1.19-4.34, $P=0.01)$, and sIL-6R (HR $1.99,95 \% \mathrm{Cl} 1.06-3.75, P=0.03$ ) were significantly associated with increased risk of early death.

\section{DISCUSSION}

The present study demonstrated that HCC patients with worse baseline liver function have elevated sTNFRII, ANGPTL4, and sgp130 levels, and lower sCD40L levels in the circulation at baseline and early during SBRT. We also noted that plasma SCD40L levels are positively associated with platelet number. SCD40L is a small protein which is primarily released by activated platelets and $\mathrm{T}$ cells ${ }^{14}$. Higher sCD40L levels have been reported in metastatic lung and nasopharyngeal cancer patients than their nonmetastatic counterparts ${ }^{15,16}$. Interestingly, elevated sCD40L levels in cancer patients as a result of platelet activation have been suggested to play an immunosuppressive role in part by upregulation the expression of PD-1 receptors on CD4+ T cells ${ }^{17}$. Ellsworth et al. ${ }^{18}$ reported that early stage non-small cell lung cancer patients undergoing hypofractionated SBRT (50-60 Gy in 10-20 fractions) have a more limited repertoire of circulating cytokines and less variability in cytokine levels at baseline and during treatment compared those receiving conventional fractionation $\mathrm{RT}$, and that $\mathrm{SCD} 40 \mathrm{~L}$ is identified as one of three cytokines (CXCL10 and macrophage inflammatory protein-1 being the remaining two cytokines) that accounts for the majority of the variability in cytokine levels seen during lung SBRT. This suggests that the systemic inflammatory/immune milieu elicited by high dose per fraction SBRT delivered focally with high conformality and sharp dose fall-off to a tumor is distinct from that produced by conventional 1.8-2 Gy per fraction radiotherapy. Our study further demonstrated that low circulating SCD40L levels in HCC patients early during SBRT are associated with increased liver toxicity and that low SCD40L levels at baseline are associated with increased risk of death at 3 months post SBRT. This is consistent with Cuneo et al. ${ }^{19}$ who previously reported that low SCD40L is associated with decline in liver function following liver SBRT in HCC patients. It is possible that the low platelet and SCD40L levels in HCC patients contribute in part to a persistent immunostimulatory environment, leading to increased liver dysfunction. A fragile pre-treatment liver is known to be more susceptible to developing radiation-induced injury ${ }^{5}$, and it is not uncommon that 

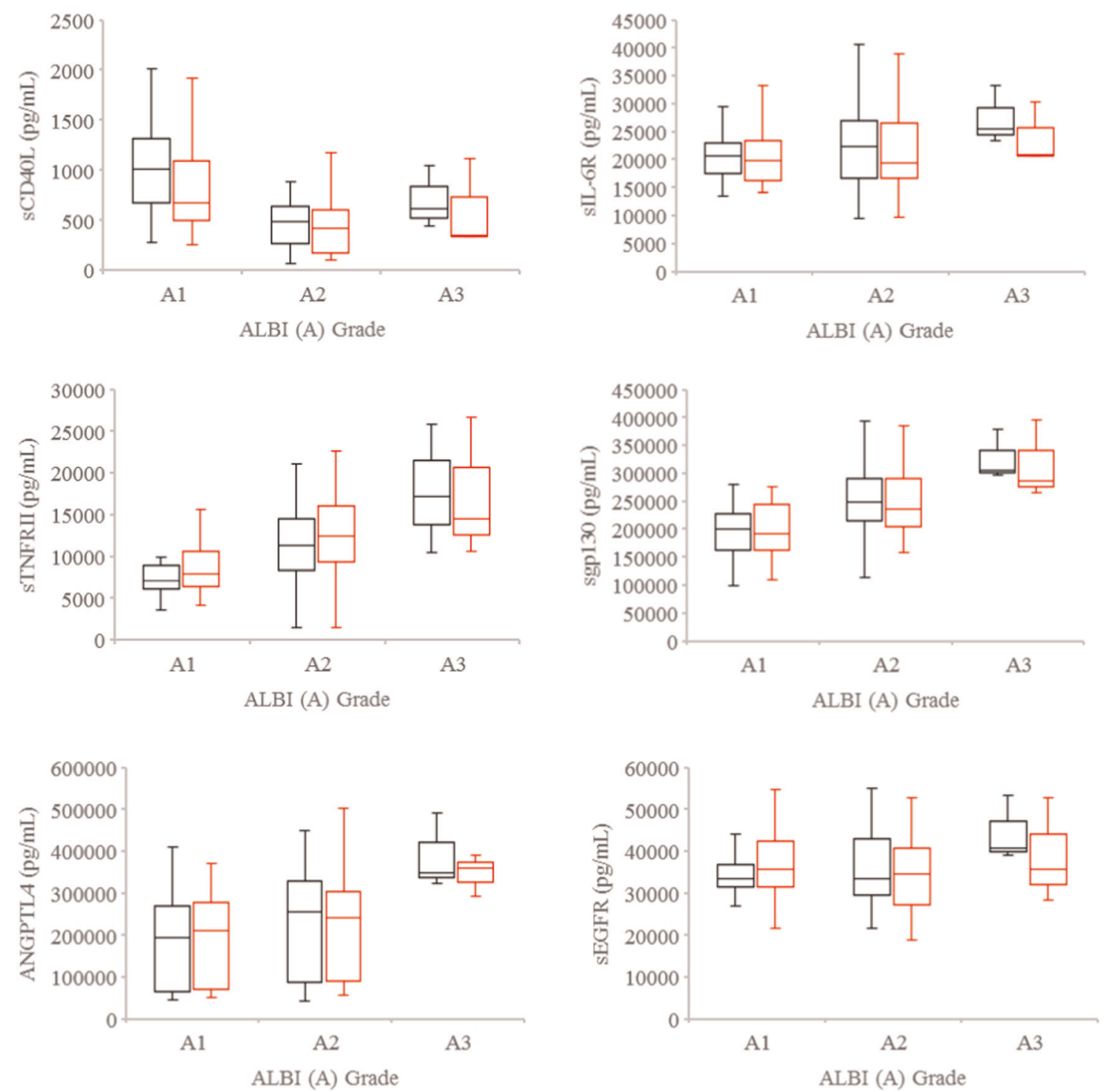

Fig. 2 Plasma levels of soluble factors by ALBI grade. Boxplots of plasma levels of sCD40L, sTNFRII, ANGPTL4, sIL-6R, sgp130, and sEGFR in ALBI grade 1, 2, and 3 hepatocellular carcinoma patients at baseline (black) and after one to two fractions of SBRT (red). Horizontal lines indicate median values, 25th-75th percentiles; whiskers indicate standard deviation.

HCC patients die of liver failure secondary to their underlying chronic liver disease rather than tumor progression. We also found that low circulating CXCL1 levels early during SBRT are also associated with increased liver toxicity at 3 months post SBRT. CXCL1, a chemotactic cytokine being secreted by nonparenchymal cells of the liver, such as hepatic stellate cells and Kupffer cells ${ }^{20}$, has been shown to promote neutrophil infiltration into HCC based on immunohistochemical studies of resected HCC tissues $^{21}$. Cui et al. ${ }^{22}$ demonstrated that HCC patients with recurrence following initial tumor resection have higher serum CXCL1 levels than those without recurrence. The mechanistic basis behind the association between low plasma CXCL1 levels early during SBRT and increased liver toxicity 3 months post SBRT seen in our study is unclear. Contrary to the Michigan ${ }^{19}$ and $\mathrm{MGH}^{23}$ studies, the present study did not find HGF levels at baseline or after one to two fractions of SBRT to be associated with liver toxicity 3 months post treatment. It is possible that the following might have contributed to the discrepancy between the Michigan ${ }^{19}$ and $\mathrm{MGH}^{23}$ data and our data: the small number of plasma samples within the range of detection of the assay in our study; the Michigan study ${ }^{19}$ consisted of patients with HCC and liver metastasis and the MGH study ${ }^{23}$ included patients with HCC, intrahepatic cholangiocarcinoma and mixed histology while our study consisted of HCC patients only; the median HGF values used as cut-off were $1.4 \mathrm{ng} / \mathrm{mL}$ (range not reported) and $2.31 \mathrm{ng} / \mathrm{mL}$ (range $1.037-8 \mathrm{ng} / \mathrm{mL}$ ) in the Michigan ${ }^{19}$ and $\mathrm{MGH}^{23}$ studies, respectively, whereas the median HGF in our study was $0.824 \mathrm{ng} /$ $\mathrm{mL}$ (range $0.16-11 \mathrm{ng} / \mathrm{mL}$ ). The definition of "higher" pretreatment HGF is relative and is likely dependent upon the patient cohort treated and the cancer subtype.

Consistent with our observation, previous studies reported higher circulating ANGPTL4 levels in patients with HCC than in those with chronic hepatitis alone and healthy controls ${ }^{24,25}$. $\mathrm{Li}$ et al. $^{24}$ demonstrated that HCC patients with intrahepatic metastasis and macrovascular invasion have higher serum ANGPTL4 levels than those without these features, suggesting a possible role of ANGPTL4 in HCC progression. ANGPTL4, a member of the angiopoietin family, is predominantly expressed in the human liver ${ }^{26}$. Preclinical and clinical data previously showed that ANGPTL4 is expressed in the hypoxic areas of human renal cell carcinoma, and promotes angiogenesis and tumorigenesis $^{27-29}$. Inflammation and infection have been shown to increase 

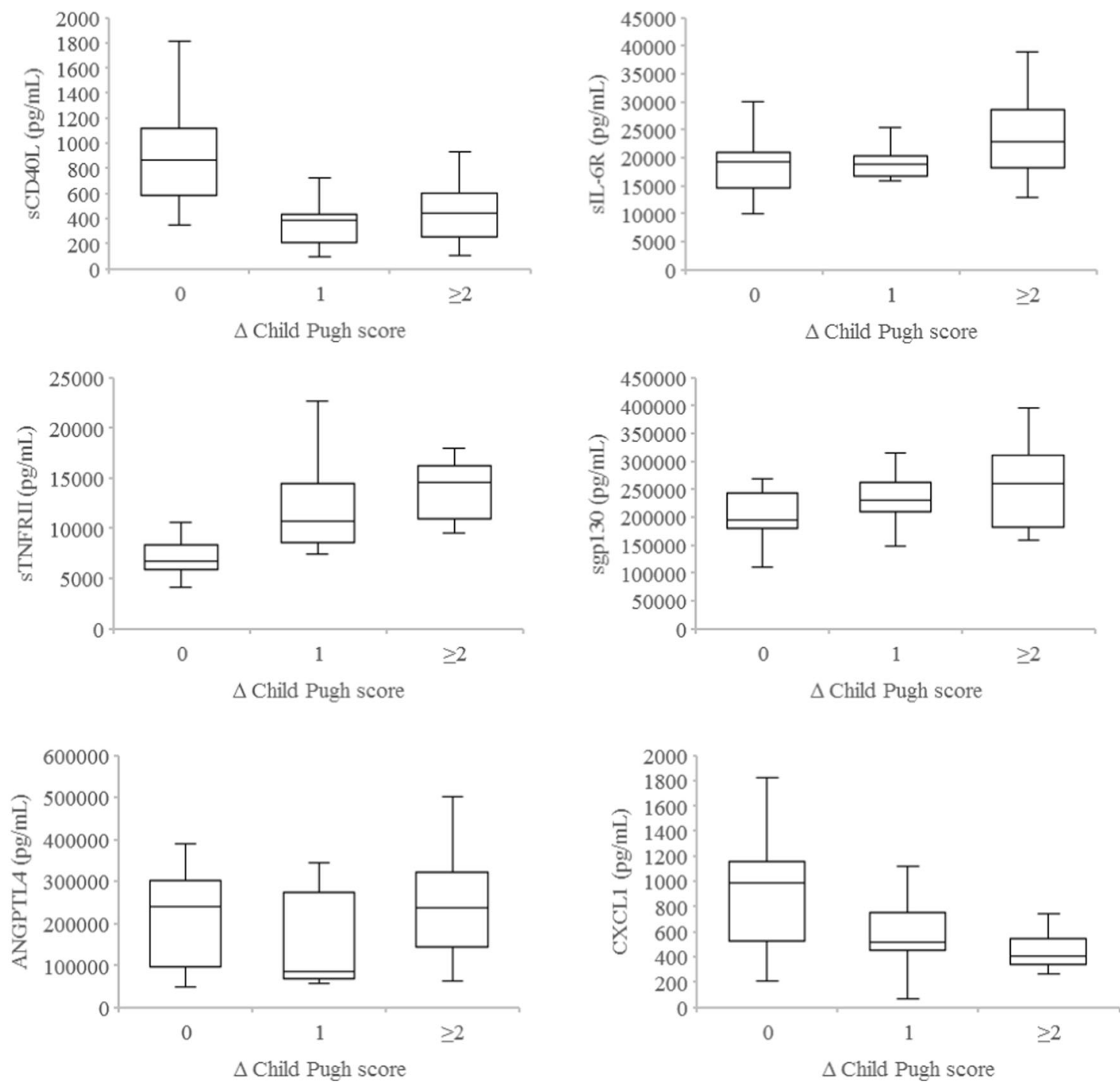

Fig. 3 Plasma levels of soluble factors by change in Child Pugh scores. Boxplots of plasma levels of sCD40L, sTNFRII, ANGPTL4, sIL-6R, sgp130, and CXCL1 following one to two fractions of SBRT in hepatocellular carcinoma patients with no change (0), 1 point increase (1), and 2 or more points increase $(\geq 2)$ in Child Pugh score at 3 months after completion of liver SBRT. Horizontal lines indicate median values, 25th-75th percentiles; whiskers indicate standard deviation.

serum ANGPTL4 levels ${ }^{30}$. The chronic hepatitis secondary to viral or non-viral causes in addition to the presence of HCC likely contributed to the high circulating ANGPTL4 levels in our patient cohort.

Our data also demonstrated the following with regard to sTNFRII: (a) HCC patients with worse liver function have significantly higher plasma levels of sTNFRII at baseline and after one to two fractions of SBRT than those with better liver function, (b) high plasma sTNFRII levels early during SBRT are associated with liver toxicity at 3 months post SBRT, and (c) high circulating sTNFRII levels at baseline and after one to two fractions of SBRT are associated with increased risk of death at 3 months after completion of SBRT. TNFRI is ubiquitously expressed, while TNFRII is predominantly expressed by immune cells and endothelial cells ${ }^{31,32}$. TNF binds to TNFRI and TNFRII and activates a complex array of signaling pathways that are involved in immune regulation, apoptosis, survival, and tumorigenesis ${ }^{32}$. sTNFRII are shed from activated immune cells and act as decoy receptors for circulating TNF, leading to inhibition of TNF's biological activity $^{31,32}$. It has been suggested that circulating sTNFRIl levels reflect TNF system activation and that sTNFRI is a better marker for liver inflammation than TNF itself because it has higher stability and longer half-life ${ }^{31,33}$. Patients with HCC and hepatitis C cirrhosis were found to have significantly higher serum sTNFRII levels compared to those with hepatitis $C$ cirrhosis alone ${ }^{34}$. It is possible that the high circulating sTNFRI levels observed in our patient cohort reflect "hyper-activation" of the TNF system secondary to chronic hepatitis and HCC, contributing to a potent proinflammatory systemic milieu and resulting in more severe liver injury and subsequent liver toxicity plus increased risk of death at 3 months after completion of SBRT. Elevated STNFRII levels was shown to be associated with lower incidence of complete response to chemotherapy \pm radiotherapy and shorter overall survival in patients with Hodgkin's lymphoma ${ }^{35}$ and nonHodgkin's lymphoma ${ }^{36}$.

Furthermore, we showed that HCC patients with worse liver function have significantly higher plasma levels of sgp130 at baseline and after one to two fractions of SBRT, and that high circulating levels of sIL-6R and sgp130 at baseline as well as high levels of sIL-6R after one to two fractions of SBRT are associated with increased risk of death at 3 months after completion of SBRT. It is well known that HCC develops in the setting of chronic liver inflammation secondary to chronic hepatitis $B / C$ infection or alcoholic/non-alcoholic steatohepatitis ${ }^{37}$. IL-6 is a pro-inflammatory 
Table 2. Association between plasma levels of AFP, ANGPTL4, CXCL1, HGF, sCD40L, sEGFR, sTNFRII, sgp130, and sIL-6R following one to two fractions of SBRT and in-field radiographic tumor response at 3 months post SBRT.

\begin{tabular}{|c|c|c|c|}
\hline \multirow[t]{2}{*}{ Plasma level of soluble factors } & \multicolumn{3}{|c|}{$\begin{array}{l}\text { In-field radiographic tumor } \\
\text { response }\end{array}$} \\
\hline & CR/PR & SD & $P$-value \\
\hline AFP & & & 0.06 \\
\hline All & $14(45 \%)$ & 17 (55\%) & \\
\hline$<$ Median & $5(29 \%)$ & $12(71 \%)$ & \\
\hline$\geq$ Median & 9 (64\%) & $5(36 \%)$ & \\
\hline ANGPTL4 & & & 0.30 \\
\hline All & $16(44 \%)$ & $20(56 \%)$ & \\
\hline$<$ Median & $10(53 \%)$ & $9(47 \%)$ & \\
\hline$\geq$ Median & $6(35 \%)$ & $11(65 \%)$ & \\
\hline CXCL1 & & & 0.40 \\
\hline All & $17(46 \%)$ & $20(54 \%)$ & \\
\hline$<$ Median & 7 (39\%) & $11(61 \%)$ & \\
\hline$\geq$ Median & $10(53 \%)$ & $9(47 \%)$ & \\
\hline HGF & & & 0.69 \\
\hline All & $10(45 \%)$ & $12(55 \%)$ & \\
\hline$<$ Median & $5(50 \%)$ & $5(50 \%)$ & \\
\hline$\geq$ Median & $5(42 \%)$ & $7(58 \%)$ & \\
\hline sCD40L & & & 0.59 \\
\hline All & $17(46 \%)$ & $20(54 \%)$ & \\
\hline$<$ Median & 7 (41\%) & $10(59 \%)$ & \\
\hline$\geq$ Median & $10(50 \%)$ & $10(50 \%)$ & \\
\hline sEGFR & & & 0.40 \\
\hline All & $17(46 \%)$ & $20(54 \%)$ & \\
\hline$<$ Median & $10(53 \%)$ & $9(47 \%)$ & \\
\hline$\geq$ Median & 7 (39\%) & 11 (61\%) & \\
\hline sTNFRII & & & 0.37 \\
\hline All & 17 (46\%) & 20 (54\%) & \\
\hline$<$ Median & 11 (52\%) & 10 (48\%) & \\
\hline$\geq$ Median & $6(38 \%)$ & $10(63 \%)$ & \\
\hline sgp130 & & & 0.63 \\
\hline All & 17 (46\%) & $20(54 \%)$ & \\
\hline$<$ Median & $9(50 \%)$ & $9(50 \%)$ & \\
\hline$\geq$ Median & $8(42 \%)$ & 11 (58\%) & \\
\hline sIL-6R & & & 0.82 \\
\hline All & 17 (46\%) & $20(54 \%)$ & \\
\hline$<$ Median & 10 (48\%) & 11 (52\%) & \\
\hline$\geq$ Median & 7 (44\%) & $9(56 \%)$ & \\
\hline
\end{tabular}

The data are the number of patients, with percentages in parentheses. $C R$ complete response, $P R$ partial response, $S D$ stable disease.

and pro-tumorigenic cytokine that exerts its effects by either classic signaling or trans-signaling ${ }^{38}$. Membrane-bound IL-6 receptors (IL-6R) are primarily expressed in hepatocytes and immune cells ${ }^{39,40}$, while membrane-bound gp130 is expressed ubiquitously by all cells. With classic signaling, two IL-6 molecules bind to two molecules of membrane-bound IL-6R, which then associate with two molecules of membrane-bound gp130, resulting in the activation of JAK/STAT3, PI3K/AKT, and RAS/ERKsignaling pathways ${ }^{38}$. Enzymatic cleavage or alternative splicing of the membrane-bound forms of IL-6R and gp130 produce their soluble counterparts, sIL-6R and sgp130, which exist naturally in
Table 3. Association between plasma levels of AFP, ANGPTL4, CXCL1, HGF, sCD40L, sEGFR, sTNFRII, sgp130, and sIL-6R and risk of death 3 months post SBRT.

\begin{tabular}{|c|c|c|c|c|c|c|}
\hline \multirow[t]{2}{*}{$\begin{array}{l}\text { Plasma levels of } \\
\text { soluble factors }\end{array}$} & \multicolumn{3}{|c|}{ At baseline } & \multicolumn{3}{|c|}{$\begin{array}{l}\text { After } 1 \text { to } 2 \text { fractions } \\
\text { of SBRT }\end{array}$} \\
\hline & $\mathrm{HR}$ & $95 \% \mathrm{Cl}$ & $P$-value & $\mathrm{HR}$ & $95 \% \mathrm{Cl}$ & $P$-value \\
\hline AFP & 2.16 & $1.03-4.54$ & $0.043^{*}$ & 2.16 & $1.03-4.54$ & $0.04^{*}$ \\
\hline ANGPTL4 & 1.10 & $0.58-2.09$ & 0.76 & 1.11 & $0.58-2.10$ & 0.75 \\
\hline CXCL1 & 0.78 & $0.41-1.46$ & 0.43 & 0.69 & $0.37-1.31$ & 0.26 \\
\hline HGF & 0.91 & $0.40-2.04$ & 0.82 & 1.15 & $0.51-2.59$ & 0.75 \\
\hline sCD40L & 0.52 & $0.27-0.99$ & $0.05^{*}$ & 0.54 & $0.29-1.05$ & 0.07 \\
\hline sEGFR & 2.61 & $1.32-5.16$ & $0.006^{*}$ & 1.80 & $0.93-3.48$ & 0.08 \\
\hline sTNFRII & 1.93 & $1.02-3.65$ & $0.04^{*}$ & 2.27 & $1.19-4.34$ & $0.01 *$ \\
\hline sgp130 & 2.19 & $1.13-4.25$ & $0.021^{*}$ & 1.71 & $0.88-3.29$ & 0.11 \\
\hline sIL-6R & 1.90 & $1.01-3.57$ & $0.05^{*}$ & 1.99 & $1.06-3.75$ & $0.03^{*}$ \\
\hline
\end{tabular}

the circulation ${ }^{41,42}$. With trans-signaling, the binding of IL-6 to sIL$6 \mathrm{R}$ is followed by binding of the IL-6:sIL-6R complex to membranebound gp130, thereby allowing cells that do not express membrane-bound IL-6R to respond to IL- $6^{38}$. Bergmann et al. ${ }^{43}$ demonstrated that IL- 6 trans-signaling, but not classic signaling, promotes the development of HCC in mice. sgp130 binds to the circulating IL-6:sIL-6R complex and acts as an inhibitor of IL- 6 transsignaling ${ }^{42}$. It has been suggested that IL- 6 classic signaling is antiinflammatory, whereas IL- 6 trans-signaling is pro-inflammatory by recruiting mononuclear cells and suppressing $\mathrm{T}$ cell apoptosis/ differentiation ${ }^{42,44}$. In HCC patients with more severe liver dysfunction, our finding of higher baseline sgp130 levels suggest an increased capacity to buffer the higher IL- 6 levels that are associated with increasing Child Pugh score ${ }^{45}$. We speculate that the higher risk of death in HCC patients with high plasma levels of sIL-6R and sgp130 at baseline and high plasma levels of sIL-6R alone early during SBRT might be attributed to decreased inhibition of IL-6:sIL-6R trans-signaling by sgp130. As a result, the balance is tipped in favor of a pro-inflammatory environment which often promotes tumor progression. Interestingly, Wierzbowska et al. ${ }^{46}$ previously reported that high circulating sIL-6R levels correlate with progression of multiple myeloma.

In conclusion, the present study provided evidence that a proinflammatory systemic environment mediated by the aforementioned soluble cytokine receptors and liver-secreted proteins exist at baseline and persist after one to two fractions of SBRT, and such systemic milieu is associated with the development of liver toxicity and increased risk of death at 3 months after completion of SBRT. This is an exploratory and hypothesis generating study, the limitations of which include small sample size, possible frequency bias, and variable time points of plasma collection after the start of SBRT (i.e., after one to two fractions instead of after one fraction for all patients). The known clinical predictor of toxicity and survival such as decline in Child Pugh score is often a comparison between pre-SBRT and 1-3 months after completion of SBRT. The highlight of our study is that changes in the levels of soluble cytokine receptors are evident early (after only one to two fractions of the planned six fractions) during treatment, thereby potentially allowing radiation oncologists to de-escalate the dose for the remaining fractions or introduce mitigating pharmacological agents and minimize toxicity later on without compromising tumor control. In this regard, we think that soluble cytokine receptor measurements complement the current clinical predictors and have the potential to further individualize radiation 
treatment. We recommend validation of the present findings in a multi-centre prospective trial that includes HCC patients who will be treated with SBRT and have their plasma samples collected for analysis at a fixed time point early during the course of treatment, e.g., after one fraction of the planned five fractions.

\section{METHODS}

Patients and treatment

Plasma samples for this study were obtained from locally advanced HCC patients who participated in two previously published prospective clinical trials (protocol \#07-0346-C) $)^{1,5}$. Written informed consent was obtained from all patients. The current study was approved by the Research Ethics Board (Oncology)/University Health Network (Toronto, ON, Canada). Briefly, eligible patients had unresectable HCC who were unsuitable for radiofrequency ablation or transcatheter arterial chemoembolization, Child Pugh A5/A6 with maximum tumor size of $15 \mathrm{~cm}$ or Child Pugh B7/B8 with maximum tumor size of $10 \mathrm{~cm}, 5$ or fewer $\mathrm{HCCs}$, and $700 \mathrm{cc}$ of uninvolved liver. The presence of portal vein thrombosis or prior therapies were permitted. The patients were treated to a total dose of 30-54 Gy in six fractions every other day. Patients who consented to participation in the aforementioned published prospective clinical trials were eligible to also consent to plasma sample collection at baseline and after having received one to two of the planned six fractions of SBRT. The collected plasma samples were stored at $-80^{\circ} \mathrm{C}$ until the time of analysis.

\section{Evaluation of clinical parameters}

Child Pugh score was determined at baseline and at 3 months post SBRT based on bilirubin, albumin, INR, and the presence/absence of ascites and encephalopathy. The change in Child Pugh score at 3 months post SBRT compared to baseline was calculated and categorized into three classes: no change or improvement, worsening of score by 1 point (e.g., from A5 to $A 6$ or $A 6$ to B7), and worsening of score by two or more points (e.g., from $A 6$ to $B 8$ or $A 5$ to $B 9$ ). ALBI score at baseline was calculated according to Johnson et al. ${ }^{7}$ : $\left(\log _{10}\right.$ bilirubin level $\left.\times 0.66\right)+($ albumin level $x-0.085$ ), whereby bilirubin was measured in $\mu \mathrm{mol} / \mathrm{L}$ and albumin level in $\mathrm{g} / \mathrm{L}$. Three prognostic groups were defined based on the following cutoffs $^{7}$ : ALBI grade $1, \leq-2.60$ l; ALBI grade $2,>-2.60$ to $\leq-1.39$; ALBI grade $3,>-1.39$. Radiographic in-field tumor response at 3 months post SBRT was assessed using RECIST version $1.1^{47}$. The development of toxicity was defined as an increase in Child Pugh score by two or more points at 3 months post SBRT in the absence of definite tumor progression according to RECIST version $1.1^{47}$.

\section{Analysis of plasma level of soluble factors}

The Milliplex Human Soluble Cytokine Receptor panel-Immunology Multiplex Assay was used to determine the plasma levels of sCD30, soluble epidermal growth factor receptor (sEGFR), soluble glycoprotein 130 (sgp130), soluble interleukin-1 receptor I and II (sIL-1RI and sIL-1RII), soluble interleukin-2 receptor alpha (sIL-2R), soluble interleukin-4 receptor (sIL-4R), soluble interleukin-6 receptor (sIL-6R), soluble receptor of advanced glycation end-products (sRAGE), soluble tumor necrosis factor receptor I and II (sTNFRI and sTNFRII), as well as soluble vascular endothelial growth receptor 1, 2, and 3 (sVEGF-R1, sVEGF-R2, and sVEGF-R3) (Millipore Sigma, Burlington, MA). The Milliplex Human Liver Protein Magnetic Bead PanelMetabolism Multiplex Assay was used to determine the plasma levels of alpha fetoprotein (AFP), angiopoietin-like 3, 4, and 5 (ANGPTL3, ANGPTL4, and ANGPTL6), fatty acid binding protein 1 (FABP1), fibroblast growth factor 10, 21, and 23 (FGF-10, FGF-21, and FGF-23), and hepatocyte growth factor (HGF) (Millipore Sigma, Burlington, MA). In addition, the Milliplex Human Cytokine/Chemokine Magnetic Bead Panel was used to evaluate the plasma levels of basic fibroblast growth factor (bFGF), chemokine C-X-C motif ligand 1 (CXCL1), eotaxin-1, interleukin 16 (IL-16), and soluble CD40 ligand (sCD40L) (Millipore Sigma, Burlington, MA). Therefore, a total of 28 plasma soluble factors were analyzed. All assays were performed according the manufacturer's instructions, and were described by Citrin et al. ${ }^{48}$. Standard curves were generated for each soluble factor with the Bio-plex manager software (Bio-Rad Laboratories, Inc., Hercules, CA) and sample concentrations were calculated from the standard curve. The sensitivity of the Milliplex assays ranged from 2.8 to $2150 \mathrm{pg} / \mathrm{ml}$ for the soluble factors being investigated. The intra-assay and inter-assay precision measured as the coefficient of variation for the analyzed soluble factors ranged from
$1.6 \%$ to $11.1 \%$ and from $4.8 \%$ to $18.9 \%$, respectively. All samples were batched by patient and a total of two plates were required to assay all samples in duplicate.

\section{Data analysis and statistics}

Statistical comparisons of continuous variables was performed using Kruskal-Wallis test. Correlation analyses of continuous and categorical data were conducted with Spearman's correlation coefficient analysis and Chisquare test, respectively. Overall survival was calculated from first day of SBRT to death or last followup. The median level of each cytokine was determined from all patients. All patients were then split into two cohorts: $<$ median level vs. $\geq$ median level of each cytokine. Associations between the levels of each cytokine and overall survival were investigated with the Cox's proportional hazard model. Statistical significance was set at $P \leq 0.05$ (two-sided). The results were considered hypothesis generating, adjustment for multiple tests was not conducted. SAS 9.4 was used for statistical analysis.

\section{Reporting summary}

Further information on experimental design is available in the Nature Research Reporting Summary linked to this paper.

\section{DATA AVAILABILITY}

The authors declare that the main data supporting the findings of this study are available within the article.

Received: 12 September 2019; Accepted: 15 May 2020; Published online: 14 July 2020

\section{REFERENCES}

1. Bujold, A. et al. Sequential phase I and II trials of stereotactic body radiotherapy for locally advanced hepatocellular carcinoma. J. Clin. Oncol. 31, 1631-1639 (2013).

2. Sanuki, N. et al. Stereotactic body radiotherapy for small hepatocellular carcinoma: a retrospective outcome analysis in 185 patients. Acta Oncol. 53, 399-404 (2014).

3. Yoon, S. M. et al. Stereotactic body radiation therapy as an alternative treatment for small hepatocellular carcinoma. PLOS ONE 8, e79854 (2013).

4. Ohri, N. et al. Local control after stereotactic body radiation therapy for liver tumors. Int. J. Radiat. Oncol. Biol. Phys. https://doi.org/10.1016/j.ijrobp.2017.12.288 (2018).

5. Culleton, S. et al. Outcomes following definitive stereotactic body radiotherapy for patients with Child-Pugh B or C hepatocellular carcinoma. Radiother. Oncol. 111, 412-417 (2014).

6. Munoz-Schuffenegger, P., Ng, S. \& Dawson, L. A. Radiation-induced liver toxicity. Semin. Radiat. Oncol. 27, 350-357 (2017).

7. Johnson, P. J. et al. Assessment of liver function in patients with hepatocellular carcinoma: a new evidence-based approach-the ALBI grade. J. Clin. Oncol. 33, 550-558 (2015).

8. Song, C. W., Kim, M.-S., Cho, L. C., Dusenbery, K. \& Sperduto, P. W. Radiobiological basis of SBRT and SRS. Int. J. Clin. Oncol. 19, 570-578 (2014).

9. Garcia-Barros, M. Tumor response to radiotherapy regulated by endothelial cell apoptosis. Science 300, 1155-1159 (2003).

10. Park, H. J., Griffin, R. J., Hui, S., Levitt, S. H. \& Song, C. W. Radiation-induced vascular damage in tumors: implications of vascular damage in ablative hypofractionated radiotherapy (SBRT and SRS). Radiat. Res. 177, 311-327 (2012).

11. Finkelstein, S. E. et al. The confluence of stereotactic ablative radiotherapy and tumor immunology. Clin. Dev. Immunol. 2011, 439752 (2011).

12. Sciulli, M. G. et al. Platelet activation in patients with colorectal cancer. Prostaglandins Leukot. Essent. Fatty Acids 72, 79-83 (2005).

13. Osada, J., Rusak, M., Kamocki, Z., Dabrowska, M. I. \& Kedra, B. Platelet activation in patients with advanced gastric cancer. Neoplasma 57, 145-150 (2010).

14. Aloui, $C$. et al. Levels of human platelet-derived soluble CD40 ligand depend on haplotypes of CD40LG-CD40-ITGA2. Sci. Rep. 6, 24715 (2016).

15. Roselli, M. et al. Soluble CD40 ligand plasma levels in lung cancer. Clin. Cancer Res. 10, 610-614 (2004).

16. Caggiari, L. et al. High serum levels of soluble CD40-L in patients with undifferentiated nasopharyngeal carcinoma: pathogenic and clinical relevance. Infect. Agent Cancer 2, 5 (2007). 
17. Huang, J. et al. Elevated serum soluble CD40 ligand in cancer patients may play an immunosuppressive role. Blood 120, 3030-3038 (2012)

18. Ellsworth, S. G. et al. Principal component analysis identifies patterns of cytokine expression in non-small cell lung cancer patients undergoing definitive radiation therapy. PLoS ONE 12, e0183239 (2017).

19. Cuneo, K. C. et al. Serum levels of hepatocyte growth factor and CD40 ligand predict radiation-induced liver injury. Transl. Oncol. 12, 889-94. (2019).

20. Marra, F. \& Tacke, F. Roles for chemokines in liver disease. Gastroenterology 147, 577-94.e1 (2014).

21. Li, L. et al. CXCR2-CXCL1 axis is correlated with neutrophil infiltration and predicts a poor prognosis in hepatocellular carcinoma. J. Exp. Clin. Cancer Res. 34, 129 (2015).

22. Cui, X. et al. Elevated CXCL1 increases hepatocellular carcinoma aggressiveness and is inhibited by miRNA-200a. Oncotarget 7, 65052-65066. (2016).

23. Hong, T. S. et al. Pretreatment plasma HGF as potential biomarker for susceptibility to radiation-induced liver dysfunction after radiotherapy. NPJ Precis. Oncol. 2, 22 (2018).

24. Li, H. et al. Hypoxia-inducible factor 1 alpha-activated angiopoietin-like protein 4 contributes to tumor metastasis via vascular cell adhesion molecule-1/ integrin $\beta 1$ signaling in human hepatocellular carcinoma. Hepatology 54, 910-919 (2011).

25. El-Shal, A. S., Zidan, H. E., Rashad, N. M. \& Wadea, F. M. Angiopoietin-like protein 3 and 4 expression 4 and their serum levels in hepatocellular carcinoma. Cytokine 96, 75-86 (2017).

26. Kim, I. et al. Hepatic expression, synthesis and secretion of a novel fibrinogen/ angiopoietin-related protein that prevents endothelial-cell apoptosis. Biochem. J. 346(Part 3), 603-10. (2000).

27. Le Jan, S. et al. Angiopoietin-like 4 is a proangiogenic factor produced during ischemia and in conventional renal cell carcinoma. Am. J. Pathol. 162, 1521-1528 (2003).

28. Zhu, P. et al. Angiopoietin-like 4 protein elevates the prosurvival intracellular $\mathrm{O} 2$ (-): $\mathrm{H} 2 \mathrm{O} 2$ ratio and confers anoikis resistance to tumors. Cancer Cell 19, 401-415 (2011).

29. Grootaert, C., Van de Wiele, T., Verstraete, W., Bracke, M. \& Vanhoecke, B. Angiopoietin-like protein 4: health effects, modulating agents and structure-function relationships. Expert Rev. Proteom. 9, 181-199 (2012).

30. Lu, B., Moser, A., Shigenaga, J. K., Grunfeld, C. \& Feingold, K. R. The acute phase response stimulates the expression of angiopoietin like protein 4. Biochem. Biophys. Res. Commun. 391, 1737-1741 (2010).

31. Moura, A. S., Carmo, R. A., Teixeira, A. L., Rocha, M. O. \& da, C. Soluble inflammatory markers as predictors of hepatocellular damage and therapeutic response in chronic hepatitis C. Braz. J. Infect. Dis. 13, 375-82. (2009).

32. Aggarwal, B. B. Signalling pathways of the TNF superfamily: a double-edged sword. Nat. Rev. Immunol. 3, 745-756 (2003).

33. Van Zee, K. J. et al. Tumor necrosis factor soluble receptors circulate during experimental and clinical inflammation and can protect against excessive tumor necrosis factor alpha in vitro and in vivo. Proc. Natl Acad. Sci. USA 89, 4845-4849 (1992).

34. Fouad, S. A., Elsaaid, N. H., Mohamed, N. A. \& Abutaleb, O. M. Diagnostic value of serum level of soluble tumor necrosis factor receptor lla in Egyptian patients with chronic hepatitis C virus infection and hepatocellular carcinoma. Hepat. Mon. 14, e19346 (2014)

35. Warzocha, K. et al. Plasma levels of tumour necrosis factor and its soluble receptors correlate with clinical features and outcome of Hodgkin's disease patients. Br. J. Cancer 77, 2357-62. (1998).

36. Heemann, C. et al. Circulating levels of TNF receptor II are prognostic for patients with peripheral T-cell non-Hodgkin lymphoma. Clin. Cancer Res. 18, 3637-3647 (2012).

37. El-Serag, H. B. \& Rudolph, K. L. Hepatocellular carcinoma: epidemiology and molecular carcinogenesis. Gastroenterology 132, 2557-2576 (2007).

38. Taniguchi, K. \& Karin, M. IL-6 and related cytokines as the critical lynchpins between inflammation and cancer. Semin. Immunol. 26, 54-74 (2014).

39. Oberg, H.-H., Wesch, D., Grüssel, S., Rose-John, S. \& Kabelitz, D. Differential expression of CD126 and CD130 mediates different STAT-3 phosphorylation in CD4+CD25- and CD25high regulatory T cells. Int. Immunol. 18, 555-63. (2006).

40. Rose-John, S. et al. Studies on the structure and regulation of the human hepatic interleukin-6 receptor. Eur. J. Biochem. 190, 79-83 (1990).
41. Chalaris, A., Schmidt-Arras, D., Yamamoto, K. \& Rose-John, S. Interleukin-6 transsignaling and colonic cancer associated with inflammatory bowel disease. Dig. Dis. 30, 492-499 (2012).

42. Scheller, J., Chalaris, A., Schmidt-Arras, D. \& Rose-John, S. The pro- and antiinflammatory properties of the cytokine interleukin-6. Biochim. Biophys. Acta 1813, 878-888 (2011).

43. Bergmann, J. et al. IL- 6 trans-signaling is essential for the development of hepatocellular carcinoma in mice. Hepatology 65, 89-103 (2017).

44. Rose-John, S. The soluble interleukin 6 receptor: advanced therapeutic options in inflammation. Clin. Pharm. Ther. 102, 591-598 (2017).

45. Soresi, M. et al. Interleukin- 6 and its soluble receptor in patients with liver cirrhosis and hepatocellular carcinoma. World J. Gastroenterol. 12, 2563-2568 (2006).

46. Wierzbowska, A., Urbańska-Ryś, H. \& Robak, T. Circulating IL-6-type cytokines and sIL-6R in patients with multiple myeloma. Br. J. Haematol. 105, 412-419 (1999).

47. Eisenhauer, E. A. et al. New response evaluation criteria in solid tumours: revised RECIST guideline (version 1.1). Eur. J. Cancer 45, 228-247 (2009).

48. Citrin, D. E. et al. Determination of cytokine protein levels in oral secretions in patients undergoing radiotherapy for head and neck malignancies. Radiat. Oncol. 7, 64 (2012).

\section{ACKNOWLEDGEMENTS}

This work was supported by the Canadian Institutes of Health Research (Grant \#202477) and National Cancer Institute of Canada (Grant \#18207).

\section{AUTHOR CONTRIBUTIONS}

All authors contributed to the acquisition, analysis, or interpretation of data, had full access to all of the data in the study and take responsibility for the integrity of the data and the accuracy of the data analysis. In addition, all authors participated in drafting and revising the work critically for important intellectual content and approved the final version.

\section{COMPETING INTERESTS}

The authors declare no competing interests.

\section{ADDITIONAL INFORMATION}

Supplementary information is available for this paper at https://doi.org/10.1038/ s41698-020-0124-z.

Correspondence and requests for materials should be addressed to L.A.D.

Reprints and permission information is available at http://www.nature.com/ reprints

Publisher's note Springer Nature remains neutral with regard to jurisdictional claims in published maps and institutional affiliations.

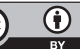

Open Access This article is licensed under a Creative Commons Attribution 4.0 International License, which permits use, sharing, adaptation, distribution and reproduction in any medium or format, as long as you give appropriate credit to the original author(s) and the source, provide a link to the Creative Commons license, and indicate if changes were made. The images or other third party material in this article are included in the article's Creative Commons license, unless indicated otherwise in a credit line to the material. If material is not included in the article's Creative Commons license and your intended use is not permitted by statutory regulation or exceeds the permitted use, you will need to obtain permission directly from the copyright holder. To view a copy of this license, visit http://creativecommons. org/licenses/by/4.0/.

(c) The Author(s) 2020 